\title{
An X-Ray Topographic Analysis of the Crystal Quality of Globally Available SiC Wafers
}

\author{
I. Brazil ${ }^{1 \text { a }}$, P.J. McNally,b ${ }^{1, \mathrm{~b}}$ N. Ren, L. O’Reilly, A. Danilewsky ${ }^{2}$, T.O. Tuomi ${ }^{3}$, A. \\ Lankinen $^{3}$, A. Säynätjaki ${ }^{3}$, R. Simon ${ }^{4}$, S. Soloviev ${ }^{5}$, L. B. Rowland ${ }^{5}$, \\ P. M. Sandvik \\ ${ }^{1}$ Research Institute for Networks \& Communications Engineering (RINCE), Dublin City University, \\ Dublin 9, Ireland \\ ${ }^{2}$ Kristallographisches Institute, Universität Freiburg, Hermann-Herder-Str. 5, Freiburg i. Br., D-79104, \\ Germany. \\ ${ }^{3}$ Helsinki University of Technology, PO Box 3500, TKK 02015, Finland. \\ ${ }^{4}$ Institut für Synchrotronstrahlung (ISS), Forschungszentrum Karlsruhe GmbH Postfach 3640, D-76021 \\ Karlsruhe, Germany. \\ ${ }^{5}$ GE Global Research, One Research Circle, Niskayuna, New York 12309, United States. \\ aibrazil@runbox.com, ${ }^{\mathrm{b}}$ Patrick.McNally@rince.ie
}

Keywords: $\mathrm{x}$-ray topography, basal plane dislocations, threading screw dislocations, large area back reflection $\mathrm{x}$-ray topography, defect analysis, defect image analysis

\begin{abstract}
.
We present herein a first comparative analysis of the quality of $50 \mathrm{~mm}$ and $75 \mathrm{~mm}$ diameter $\mathrm{SiC}$ wafers, purchased directly from vendors across the world, types including the most widely available configurations. Large Area White Beam Synchrotron Back Reflection X-Ray Topography was used to analyse selected $\sim 1 \mathrm{~cm}^{2}$ regions at various locations on up to 10 different bulk $\mathrm{SiC}$ wafers.

The study concentrated particularly on the density and distribution of threading screw dislocations (TSDs). We also examined all wafers for basal plane dislocation (BPDs) densities and distributions.

Alarmingly large variation in wafer quality was observed. TSD densities vary from a minimum of $0 \mathrm{~cm}^{-}$

2 (in a-plane material) to values as large as over $2,000 \mathrm{~cm}^{-2}$ on some n-type $4 \mathrm{H}-\mathrm{SiC}$ wafers. TSD densities on individual wafers can also vary by similar magnitudes, e.g. $500 \mathrm{~cm}^{-2}$ to $2,500 \mathrm{~cm}^{-2}$ on two regions only $2 \mathrm{~cm}$ apart on a $50 \mathrm{~mm}$ diameter wafer.

Computer-based image process analysis was used to present a statistical analysis of the distributions of defects. For example algorithms created in MATLAB®, Image Processing Toolbox, isolated possible TSD locations allowing rapid counting to be performed. These counts were confirmed by manual counting of selected unmodified images.
\end{abstract}

\section{Introduction}

Silicon Carbide ( $\mathrm{SiC}$ ) has attracted a lot of interest as of late. Benefits include the possibility of creating semiconductor devices which can operate at extremely high temperatures, its wide band gap and its large electrical breakdown field [1] together with its radiation hard abilities allow its use in harsh environments. SiC however is quite prone to defects [2,4]. The production of extremely high intensity low divergence $\mathrm{x}$-ray beams at synchrotron sources provides for an ideal for examination technique via $\mathrm{x}$-ray topography [2,3,5]. Imaging was undertaken at the HASYLAB and ANKA Synchrotrons in Hamburg and Karlsruhe, respectively, in Germany. Wafer types included the most widely available configurations including n-type $4 \mathrm{H}-\mathrm{SiC}$, p-type $4 \mathrm{H}-\mathrm{SiC}$, n-type $6 \mathrm{H}-\mathrm{SiC}$, p-type $6 \mathrm{H}-$ $\mathrm{SiC}$, a-plane $4 \mathrm{H}-\mathrm{SiC}$ and a $6 \mathrm{H}-\mathrm{SiC}$ Lely reference platelet. 


\section{Algorithms implemented for processing.}

We developed two semi-automatic defect detection and counting algorithms using MATLAB®, one for BPDs and one for TSDs allowing for semi-automatic detection/counting in both. Preliminary analysis revealed TSDs typically had pixel intensities greater than the surrounding image in topography and that TSDs tended to cluster around similar pixel values. The algorithm developed thus for TSDs was based on intensity difference. A rough outline of the implementation follows.

The majority of the images could be broken down to 2 types of pixel intensity ranges (a High and a Low). Type 1 represents high pixel intensity values these being extremely 'bright' TSDs. A high value threshold, at an appropriate value, extracted out high intensity TSDs (very white TSDs). Type 2 (Low) threshold, represented a pixel range of values requiring a dual threshold [6] at the appropriate range. Thus two output threshold images were produced. Thresholds carry a lot of unwanted information; removal of this was based on the average size of TSDs observed and carried out using morphological techniques to remove pixel clusters not representative of TSDs. Perimeter finding (with noise removed automatically) was used for reference. Dilation of the High Processed output image from above and subtraction of this from the Low Processed image allows detection and removal of coinciding TSDs by using morphological techniques. Where a TSD had been found in the high or low find, a black circle of appropriate diameter was used to automatically represent the find. This black circle was then added back to the original image to represent a possible location of a TSD, thus allowing batch processing.

If required these identified locations could be manually checked with speed. Thus a semi-automatic method was developed for counting of TSD numbers. In Figure 1 we can see the raw input image Fig. 1(a), and on the resultant output 'high' find image Fig. 1(b). Black circles representing surrounding possible locations of high value TSDs can be seen. Similar images were available for "low". Both counts could be combined to have an overall number of TSDs. As a check on the process, manual counting was performed on selected unmodified images. Average error was determined to be approximately 20 percent for the selected images with the process always over counting

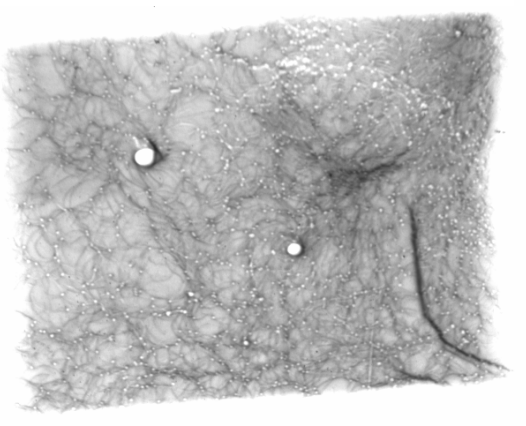

(a) Input Image

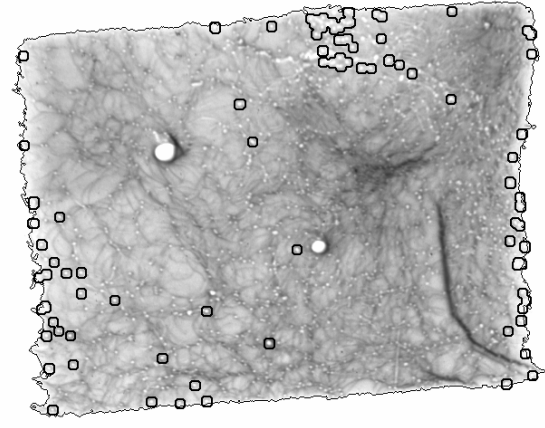

(b) Output High Intensity Image

Figure 1

The BPD algorithm used a separate algorithm on the raw images. The algorithm was developed as follows. Lines representing the Basal Plane Dislocations in the images were noticed to be of varying intensity even along the same line, but there always existed a boundary difference between the line and the surrounding image. The first step took advantage of adaptive histogram equalization (AHE) to highlight more clearly the background lines representing the BPDs. The 'vein' type structure of the BPD running through the image can be clearly seen in Fig. 2(b). A simple edge detection method was implemented in the algorithm to extract out as many of the lines as possible from the image. This was followed by simple morphological techniques to represent each BPD as a single pixel width line. This process can be seen in Fig. 2(c). 


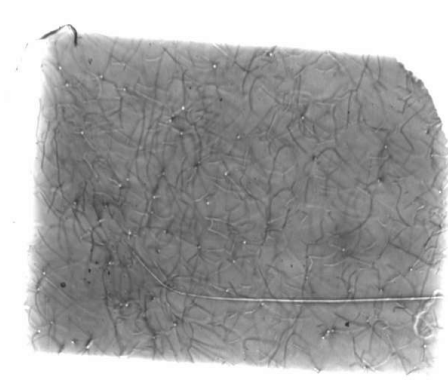

(a) Original Image

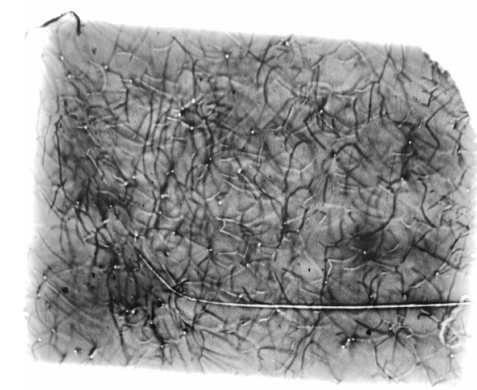

(b) AHE

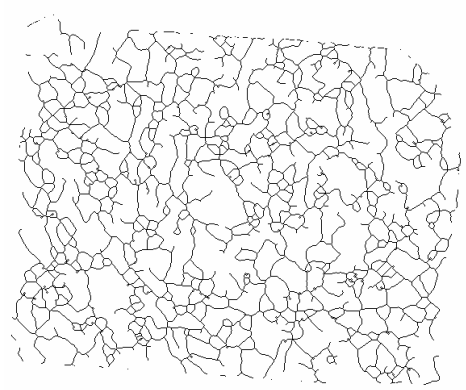

(c) BPD Output

Figure 2

In certain instances output image required manual cleaning by simple erasing of the additional lines found to be unrepresentative of BPDs. This was the only manual step. BPDs could then be automatically counted and values outputted automatically representing their number. Counting was achieved by summing the black pixels in Fig. 2(c). Each image had the number of pixels previously counted on longest edge and compared to the physical dimensions for that image. This gave a representation for the physical dimensions of each pixel, an approximate area of pixels representing BPD's was thus established for each image.

\section{Results}

\section{Average TSD Density per wafer}

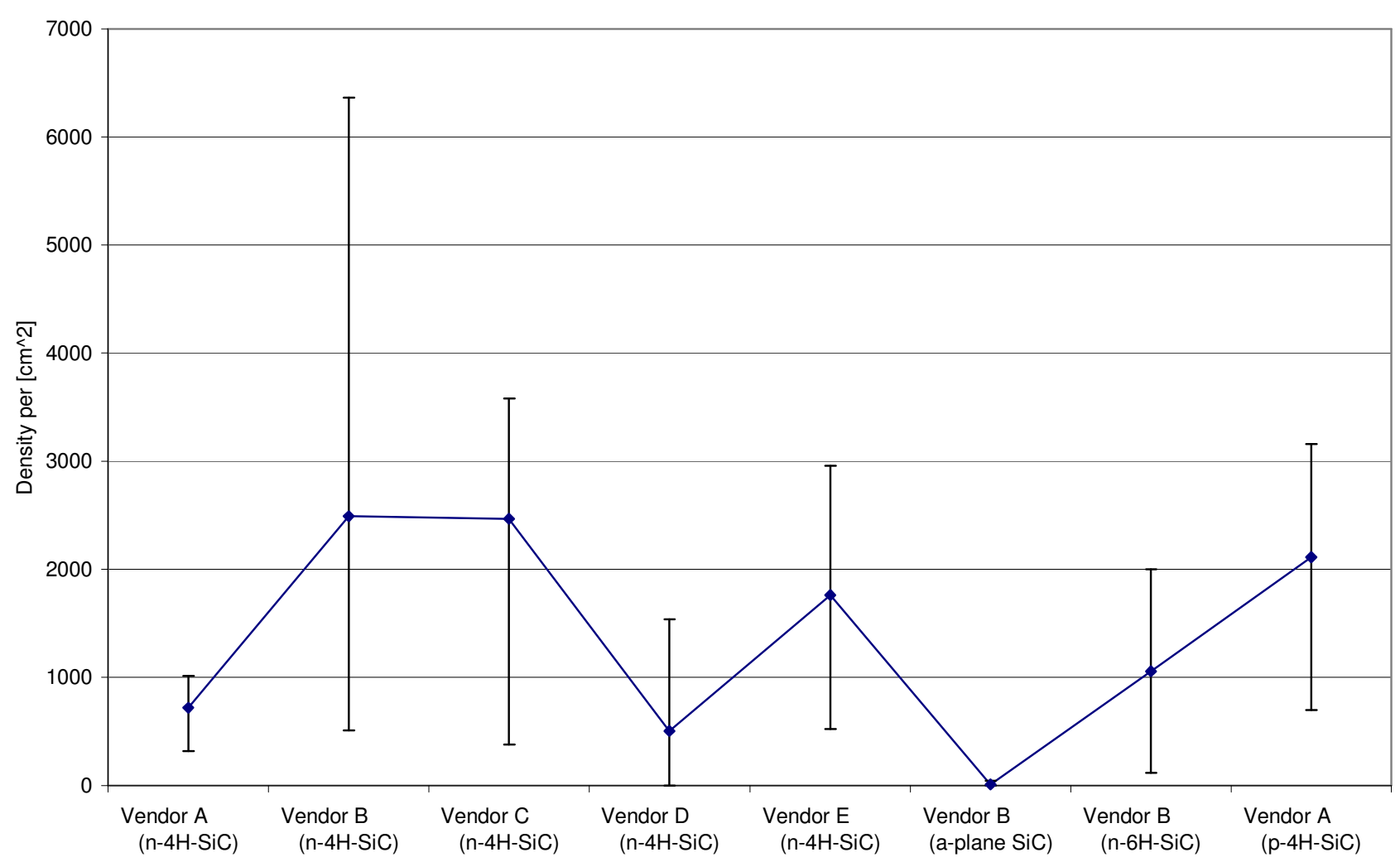

Figure 3 - Graph showing Average TSD Density

Fig. 3, indicates the observed variation of TSD densities for a selection of whole $50 \mathrm{~mm}$ and $75 \mathrm{~mm}$ diameter SiC wafers whilst Fig. 4, represents the BPD densities. The "error bars" indicate the measured variation as observed at different locations on these wafers, the average TSD (or BPD) density being indicated by the filled circle for each wafer. Penetration depth was not taken into account 
in the above density calculations for either Fig. 3, or Fig. 4. The large variation in TSD densities on both the same wafer and across wafers is extremely apparent.

Average BPD per Wafer

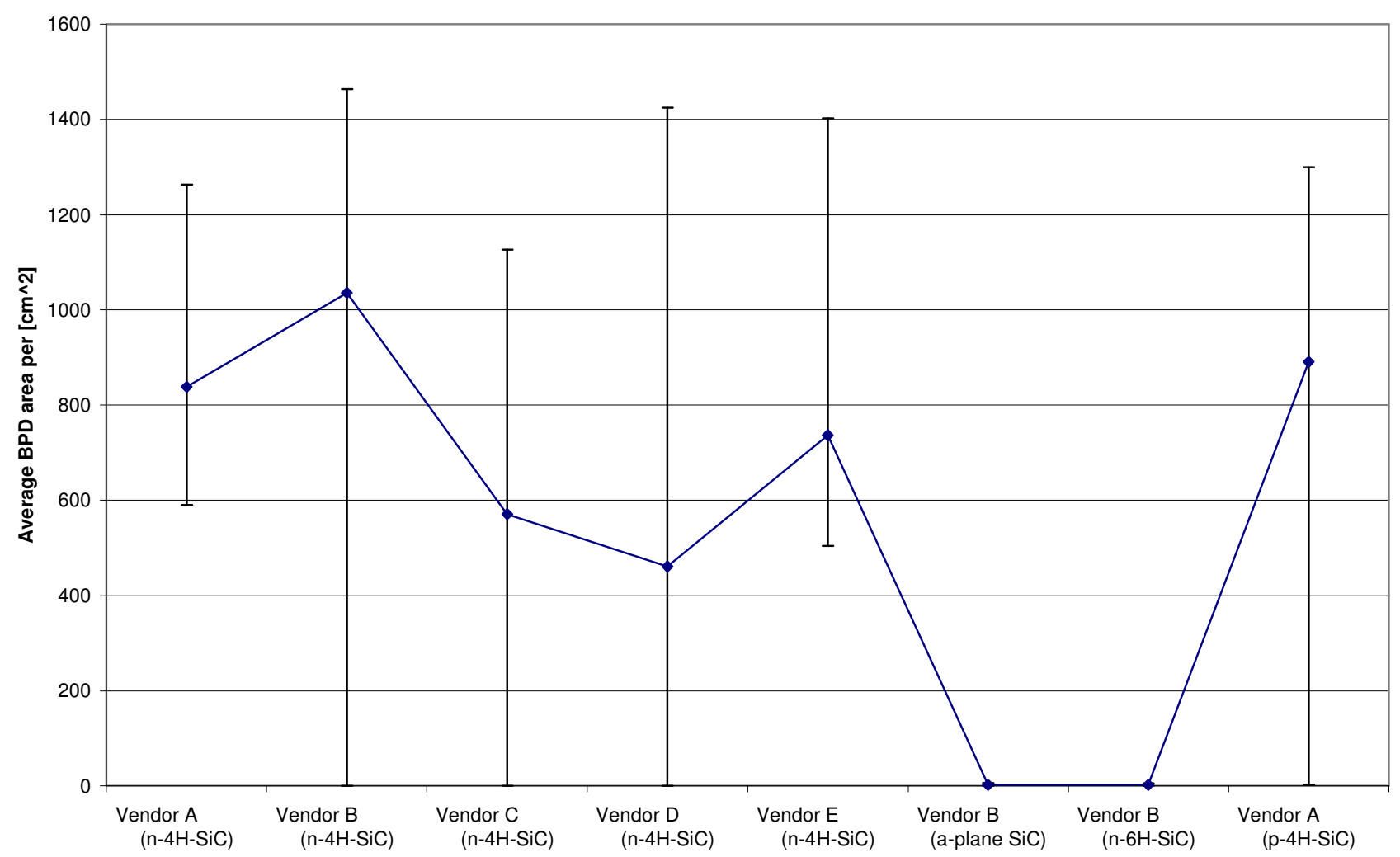

Figure 4 - Average BPD per wafer

The variation in different positions on same wafer for BPDs is astonishingly large (represented by the error bars). As a measure of quality the a-plane $\mathrm{SiC}$ has the best results. The $6 \mathrm{H}-\mathrm{SiC}$ comes in next best from above; both the $6 \mathrm{H}$ and a-plane $\mathrm{SiC}$ originate from Vendor $\mathrm{B}$. The $\mathrm{n}-4 \mathrm{H}$ and $\mathrm{p}-4 \mathrm{H}$ wafers have some of the highest average values together with the largest ranges. Vendor A produced the best quality (TSD) in the $\mathrm{n}-4 \mathrm{H}$ variety. For BPDs all $4 \mathrm{H}$ type wafers had relatively poor quality. Further work on these results is ongoing together with improvements to the accuracy of the algorithm.

\section{References:}

[1] T. Ohno, H. Yamaguchi, S. Kuroda, K. Kojima, T. Suzuki and K. Arai: Journal of Crystal Growth Vol. 271 (2004), p1

[2] T. Ohno, H. Yamaguchib, S. Kurodac, K. Kojimaa, T. Suzukia and K. Araic: Journal of Crystal Growth Vol. 260 (2004), p209

[3] Michael Dudley, XianRong Huang and William M Vetter: J. Phys. D: Appl. Phys. Vol. 36 (2003), pA30

[4] Seoyong Ha, Noel T. Nuhfer, Gregory S. Rohrer, Marc De Graef and Marek Skowronski: Journal of Crystal Growth Vol. 220 (2000), p308

[5] D.K. Bowen and Brian K. Tanner, in: High Resolution X-Ray Diffractometry Topography, Taylor and Francis Inc. Publishing (2001)

[6] P.F. Whelan and D.Molloy, in: Machine Vision Algorithms in Java Techniques and Implementation, Springer-Verlab Publishing (2002) 